\title{
Endemic Acinetobacter baumannii in a New York Hospital
}

\author{
Scott A. Weisenberg ${ }^{1 *^{\alpha}}$, Audrey N. Schuetz ${ }^{2}$, Elizabeth A. Alexander ${ }^{1}$, Brain Eiss ${ }^{1}$, Maryam Behta ${ }^{3}$, Lisa \\ Saiman $^{4,5}$, Davise H. Larone ${ }^{2}$, Stephen G. Jenkins ${ }^{2}$, Kyu Y. Rhee'
}

1 Department of Medicine, Weill Cornell Medical College, New York, New York, United States of America, 2 Department of Pathology, Weill Cornell Medical College, New York, New York, United States of America, 3 Department of Information Services, New York-Presbyterian Healthcare Medical Centers, New York, New York, United States of America, 4 Department of Pediatrics, Columbia University, New York, New York, United States of America, 5 Department of Infection Prevention and Control, New YorkPresbyterian Hospital, New York, New York, United States of America

\begin{abstract}
Background: Acinetobacter baumannii is an increasingly multidrug-resistant (MDR) cause of hospital-acquired infections, often associated with limited therapeutic options. We investigated $A$. baumannii isolates at a New York hospital to characterize genetic relatedness.

Methods: Thirty A. baumannii isolates from geographically-dispersed nursing units within the hospital were studied. Isolate relatedness was assessed by repetitive sequence polymerase chain reaction (rep-PCR). The presence and characteristics of integrons were assessed by PCR. Metabolomic profiles of a subset of a prevalent strain isolates and sporadic isolates were characterized and compared.

Results: We detected a hospital-wide group of closely related carbapenem resistant MDR A. baumannii isolates. Compared with sporadic isolates, the prevalent strain isolates were more likely to be MDR $(p=0.001)$. Isolates from the prevalent strain carried a novel Class I integron sequence. Metabolomic profiles of selected prevalent strain isolates and sporadic isolates were similar.

Conclusion: The A. baumannii population at our hospital represents a prevalent strain of related MDR isolates that contain a novel integron cassette. Prevalent strain and sporadic isolates did not segregate by metabolomic profiles. Further study of environmental, host, and bacterial factors associated with the persistence of prevalent endemic A. baumannii strains is needed to develop effective prevention strategies.
\end{abstract}

Citation: Weisenberg SA, Schuetz AN, Alexander EA, Eiss B, Behta M, et al. (2011) Endemic Acinetobacter baumannii in a New York Hospital. PLoS ONE 6(12): e28566. doi:10.1371/journal.pone.0028566

Editor: Brad Spellberg, Los Angeles Biomedical Research Institute, United States of America

Received August 23, 2011; Accepted November 10, 2011; Published December 13, 2011

Copyright: (c) 2011 Weisenberg et al. This is an open-access article distributed under the terms of the Creative Commons Attribution License, which permits unrestricted use, distribution, and reproduction in any medium, provided the original author and source are credited.

Funding: This investigation was supported by grant KL2RR024997 of the Clinical and Translation Science Center at Weill Cornell Medical College (SAW). The funders had no role in study design, data collection, and analysis, decision to publish, or preparation of the manuscript. No additional external funding was received for this study.

Competing Interests: The authors have read the journal's policy and have the following conflicts: Scott Weisenberg has received an investigator initiated grant from Merck for an unrelated study (related to international travel and importation of ESBL producing bacteria). Kyu Rhee has received support for unrelated studies on Enterococci from Cubist. This does not alter the authors' adherence to all the PLoS ONE policies on sharing data and materials.

* E-mail: scottweisenberg@gmail.com

Current address: Alta Bates Summit Medical Center, Oakland, California, United States of America

\section{Introduction}

Acinetobacter baumannii is a nonfermentative, nonmotile, oxidasenegative, catalase positive gram-negative bacillus [1], and is an increasing cause of intensive care unit and hospital-associated infections [2]. Though the attributable mortality of $A$. baumannii infection continues to be debated [1], clinicians increasingly are confronted with infections with few or no antibiotic options. For this reason, A. baumannii has been identified as a particularly problematic pathogen by the Antimicrobial Availability Task Force of the Infectious Disease Society of America [3].

A. baumannii is now prevalent throughout New York City hospitals [4]. Like most hospitals in the area, NewYorkPresbyterian Hospital/Weill Cornell Medical College (NYPH/ WCMC) has experienced an increase in A. baumannii infection and colonization during the last 10 years, though the relatedness of the isolates was largely unknown. We therefore studied the genetic relatedness of the hospital wide $A$. baumannii population at NYPH/ WCMC during a period when the incidence and prevalence were stable. We subsequently compared the microbiologic, genetic, and metabolic characteristics of the prevalent endemic strain and sporadic isolates.

\section{Materials and Methods}

\section{Isolate Selection}

Institutional Review Board approval with waiver of informed consent was obtained. Isolates cultured at least 48 hours after admission between February and December 2008 were prospectively obtained from the Clinical Microbiology Laboratory, as 
were results of species identification and antimicrobial susceptibility testing. VITEK2 GN13 cards (bioMérieux Inc., Durham, NG) were used to identify members of the $A$. calcoaceticus- A. baumannii complex, and to assess antimicrobial susceptibility. In addition, Etests ( $\mathrm{AB}$ bioMérieux, Durham, $\mathrm{NC}$ ) were used to assess susceptibility to polymyxin $\mathrm{B}$ and tigecycline when requested by treating clinicians, or when the isolate was multidrug resistant. Multidrug-resistance (MDR) was defined as susceptibility to $\leq 1$ tested antibiotic, excluding tigecycline and polymyxin B. No additional antimicrobial resistance testing was performed beyond what had been done for clinical purposes. Nursing unit and date of culture were obtained from the electronic medical record. Available A. baumannii isolates were intermittently collected from the microbiology laboratory during the study period. A total of 103 A. baumannii isolates from 50 patients were available, and only the first isolate per patient was used for this study. The total number of patients with an A. baumannii culture during the study period was 128 . Isolates from 78 patients were unavailable due to sporadic collection of isolates. A convenience sample of 30 study isolates was selected from among the collected isolates to represent the largest number of intensive care units (ICUs) and wards in order to characterize the hospital wide A. baumannii population. The 19 excluded first isolates were from ICU patients whose ICU was already represented in the study database (12), non-ICU patients with contaminated plates (3), and from patients in hospital for less than 48 hours at the time of collection (4). The 12 excluded ICU isolates all contained the same integron pattern as the prevalent endemic isolates identified in the study. One of the 4 isolates collected in the first 48 hours was included (WC-31) since they had been transferred from an outside hospital and thus provided an opportunity to compare hospital strains. Only one isolate per patient was studied.

\section{Characterization of Isolates}

All study isolates underwent Repetitive PCR (Rep-PGR) testing and Class I Integron analysis. After the initial analysis showed a prevalent endemic group of related isolates and additional sporadic isolates, we made the simplifying assumption that isolates with closely linked Rep-PCR results were of the same species and Multi Locus Sequence Typing (MLST) type. Thus, OXA-51-like gene amplification was done on all sporadic and the closest 5 prevalent endemic isolates, and MLST typing was done on a single prevalent endemic isolate. Metabolome analysis was performed on a subset of prevalent and endemic isolates. DNA templates and primers were prepared as described by Levasque et al. [5] with the exception that 3-5 colonies were dissolved in $100 \mu \mathrm{l}$ of nuclease- free water, and selective antibiotics were not used. PCR amplification conditions for the 5'CS -3'CS integron analysis were as described by MartinezFreijo et al. [6], with a two minute annealing time. A second PCR with non-overlapping primers was used to verify the integron contents of the prevalent endemic strain [7]. A. baumannii, OXA-51like chromosomal genes were amplified from isolates WC 1-12 using previously described primers [8]. High Fidelity PCR Master mix was used for all reactions (Roche, Indianapolis, IN). PCR product nucleotide sequences were determined by Macrogen USA (Rockville, MD). Sequences were compared to known sequences using the Basic Local Alignment Search Tool (BLAST). MLST was performed using a selected isolate from the prevalent endemic strain and compared to the Pasteur MLST site (http://www.pasteur.fr/ recherche/genopole/PF8/mlst/Abaumannii.html).

\section{Repetitive-PCR typing}

DNA was extracted using the UltraClean ${ }^{\mathrm{TM}}$ microbial DNA isolation kit (MO BIO Labs, Inc., Solana Beach, CA). Rep-PGR was performed using the DiversiLab system (bioMérieux Inc., Durham, NG). The model 2100 bioanalyzer and LabChip reagents (Agilent Technologies Inc., Palo Alto, CA) were used for DNA separation. The DiversiLab system created dendrograms, scatterplots, electropherograms, and virtual gel images for data analysis. Similarity criteria were followed as per Saeed $[9]$.

\section{Metabolome Analysis}

Differences in the metabolic properties of selected isolates were studied by examining individual metabolites of interest and global metabolic profiles. Biological replicates of A. baumannii isolates were cultivated in phosphate-M9 minimal media [10] and grown for metabolomic profiling on phosphate-M9 agar [11]. Bacterial isolates were harvested during mid-logarithmic phase of growth, and metabolites were isolated as described previously [12]. Variations in biomass were normalized for all isolates by analysis of residual protein content [13]. An Agilent Accurate Mass 6220 time-of-flight mass spectrometer coupled to an Agilent 1200 liquid chromatography system was used as described previously [12]. Data were collected in centroid mode in $2 \mathrm{GHz}$ (extended dynamic range) mode. Detected masses were deemed identified metabolites on the basis of unique accurate mass retention times which confirmed to the expected metabolites' mass and that of accompanying isotopomers [14]. Deconvolution of mass spectra was performed using the Agilent MassHunter Qualitative Analysis molecular feature extraction algorithm which identifies individual metabolites using chromatographically covariant ion families which conform to specific empirical formulae [14]. The normalized ion counts (in triplicate) of 3 prevalent endemic strain isolates were compared to the ion counts of 3 sporadic isolates (in triplicate), and compared using Stata version 10.0 by Wilcoxon Rank-Sum test. Additional statistical analyses used GeneSpring MS software to perform principle components analysis (PCA) and hierarchical clustering analysis (HCA).

\section{Results}

\section{Study Isolates and Antimicrobial Susceptibility}

Thirty isolates from unique patients were studied (Table 1). The study isolates represented $24.2 \%$ of the 128 A. baumannii isolates cultured from unique patients during that period. Patient locations at the time of culture were geographically widespread, including seven ICUs (5 floors) and seven non-ICU nursing units (5 floors) over a 9 month period. An additional isolate (WC-31), cultured on the first day of hospitalization from a patient transferred to NYPH/WCMC from another hospital in Brooklyn in New York City, was also included in the rep-PCR and integron analyses.

The majority $(70 \%)$ of the isolates in this study were multidrugresistant. The proportion of isolates susceptible to tested antimicrobial agents included ampicillin-sulbactam (33\%), imipenem or meropenem $(23 \%)$, cefepime $(17 \%)$, gentamicin $(27 \%)$, tobramycin $(33 \%)$, amikacin $(33 \%)$, trimethoprim-sulfamethoxazole $(17 \%)$, and levofloxacin $(20 \%)$. In contrast, 20 of the 22 $(91 \%)$ isolates tested were susceptible to polymyxin B $(\mathrm{MIC} \leq 2 \mu \mathrm{g} / \mathrm{mL}$ ), though the MICs for eight were between 1.5 and $2 \mu \mathrm{g} / \mathrm{mL}$. Tigecycline MICS were $\leq 2 \mu \mathrm{g} / \mathrm{mL}$ for 11 of the $25(44 \%)$ isolates tested. The chromosomal A. baumannii gene blaOXA-51-like carbapenemase gene [8] could not be amplified from four isolates (WC 1-4), suggesting that they belonged to other A. calcoaceticus-A. baumannii complex species. Pending further identification, these isolates are considered " $A$. baumannii" in this study. 
Table 1. Study Isolates.

\begin{tabular}{|c|c|c|c|c|c|c|c|c|c|c|c|c|c|}
\hline$w C$ & $P T$ & Date & FLOOR & $A / S$ & Carb & Cef & Gent & Amik & $S / T$ & LEVO & $P M$ & Tig & SOURCE \\
\hline 1 & $68 \mathrm{~F}$ & $9 / 5$ & A ICU & s & s & s & S & s & $\mathrm{R}$ & S & & & RESP \\
\hline 2 & $48 \mathrm{M}$ & $2 / 29$ & A ICU & $\mathrm{R}$ & $\mathrm{R}$ & $\mathrm{R}$ & $\mathrm{R}$ & $\mathrm{R}$ & $\mathrm{R}$ & 1 & 0.8 & 4 & RESP \\
\hline 3 & $12 \mathrm{~F}$ & $9 / 26$ & B ICU & s & $\mathrm{s}$ & s & $\mathrm{s}$ & s & $\mathrm{s}$ & S & & & RESP \\
\hline 4 & $18 \mathrm{~F}$ & $8 / 3$ & B ICU & s & S & s & $\mathrm{S}$ & s & S & $\mathrm{s}$ & & 0.5 & RESP \\
\hline 5 & $58 \mathrm{M}$ & $10 / 8$ & CICU & $\mathrm{s}$ & s & 1 & S & $\mathrm{R}$ & $\mathrm{R}$ & 1 & & & RESP \\
\hline 6 & $40 \mathrm{~F}$ & $7 / 30$ & B WARD & s & $\mathrm{s}$ & s & $\mathrm{s}$ & S & s & $\mathrm{s}$ & & & URINE \\
\hline 7 & $79 F$ & $8 / 24$ & D ICU & $\mathrm{s}$ & $\mathrm{S}$ & s & $\mathrm{s}$ & S & S & S & & & RESP \\
\hline 8 & $76 \mathrm{M}$ & $9 / 22$ & E ICU & S & $\mathrm{R}$ & $\mathrm{R}$ & $\mathrm{R}$ & $\mathrm{R}$ & $\mathrm{R}$ & $\mathrm{R}$ & 1 & 12 & RESP \\
\hline 9 & $76 \mathrm{~F}$ & $10 / 26$ & C ICU & $\mathrm{R}$ & $\mathrm{R}$ & $\mathrm{R}$ & $\mathrm{R}$ & 1 & $\mathrm{R}$ & 1 & 0.75 & 4 & RESP \\
\hline 10 & $71 M$ & $11 / 7$ & D WARD & $\mathrm{R}$ & $\mathrm{R}$ & $\mathrm{R}$ & $\mathrm{R}$ & $\mathrm{R}$ & $\mathrm{R}$ & $\mathrm{R}$ & 1 & 12.5 & BLOOD \\
\hline 11 & $3 \mathrm{~F}$ & $8 / 8$ & B ICU & $\mathrm{R}$ & $\mathrm{R}$ & $\mathrm{R}$ & $\mathrm{R}$ & $\mathrm{R}$ & $\mathrm{R}$ & 1 & 1.5 & 3 & RESP \\
\hline 12 & $6 \mathrm{M}$ & $9 / 15$ & A ICU & $\mathrm{R}$ & $\mathrm{R}$ & $\mathrm{R}$ & $\mathrm{R}$ & S & $\mathrm{R}$ & 1 & 1.5 & 3 & URINE \\
\hline 13 & $78 \mathrm{~F}$ & $7 / 13$ & D WARD & s & $\mathrm{R}$ & $\mathrm{R}$ & $\mathrm{R}$ & $\mathrm{R}$ & $\mathrm{s}$ & S & 1 & 3 & URINE \\
\hline 14 & $46 \mathrm{~F}$ & $6 / 24$ & D ICU & $\mathrm{R}$ & $\mathrm{R}$ & $\mathrm{R}$ & $\mathrm{R}$ & $\mathrm{R}$ & $\mathrm{R}$ & $\mathrm{R}$ & 4 & 2 & BLOOD \\
\hline 15 & $67 M$ & $9 / 20$ & E ICU & $\mathrm{R}$ & $\mathrm{R}$ & $\mathrm{R}$ & $\mathrm{R}$ & 1 & $\mathrm{R}$ & $\mathrm{R}$ & 1 & 2 & RESP \\
\hline 16 & $67 \mathrm{M}$ & $9 / 2$ & A WARD & $\mathrm{R}$ & $\mathrm{R}$ & $\mathrm{R}$ & S & 1 & $\mathrm{R}$ & 1 & 1.5 & 3 & URINE \\
\hline 17 & $34 \mathrm{M}$ & $11 / 23$ & A ICU & $\mathrm{R}$ & $\mathrm{R}$ & $\mathrm{R}$ & $\mathrm{R}$ & $\mathrm{R}$ & $\mathrm{R}$ & $\mathrm{R}$ & 2 & 4 & RESP \\
\hline 18 & $71 \mathrm{~F}$ & $10 / 26$ & A ICU & $\mathrm{R}$ & $\mathrm{R}$ & $\mathrm{R}$ & $\mathrm{R}$ & 1 & $\mathrm{R}$ & 1 & 0.5 & 2 & BLOOD \\
\hline 19 & $62 M$ & $12 / 6$ & D ICU & R & $\mathrm{R}$ & $\mathrm{R}$ & $\mathrm{R}$ & $\mathrm{R}$ & $\mathrm{R}$ & $\mathrm{R}$ & 0.5 & 6 & RESP \\
\hline 20 & $87 \mathrm{~F}$ & $11 / 5$ & F WARD & $\mathrm{R}$ & $\mathrm{R}$ & $\mathrm{R}$ & $\mathrm{R}$ & $\mathrm{R}$ & $\mathrm{R}$ & $\mathrm{R}$ & 0.5 & 8 & BLOOD \\
\hline 21 & $78 \mathrm{M}$ & $11 / 30$ & E WARD & $\mathrm{R}$ & $\mathrm{R}$ & $\mathrm{R}$ & $\mathrm{R}$ & $\mathrm{R}$ & $\mathrm{R}$ & $\mathrm{R}$ & 2 & 2 & BLOOD \\
\hline 22 & $65 \mathrm{~F}$ & $9 / 20$ & E ICU & $\mathrm{R}$ & $\mathrm{R}$ & $\mathrm{R}$ & $\mathrm{R}$ & S & $\mathrm{R}$ & $\mathrm{R}$ & 0.75 & 2 & BLOOD \\
\hline 23 & $43 \mathrm{M}$ & $8 / 18$ & A ICU & $\mathrm{R}$ & $\mathrm{R}$ & $\mathrm{R}$ & $\mathrm{R}$ & S & $\mathrm{R}$ & I & 32 & 4 & RESP \\
\hline 24 & $76 \mathrm{M}$ & $8 / 10$ & E ICU & $\mathrm{R}$ & $\mathrm{R}$ & $\mathrm{R}$ & $\mathrm{R}$ & $\mathrm{R}$ & $\mathrm{R}$ & 1 & 0.5 & 2 & BLOOD \\
\hline 25 & $20 \mathrm{M}$ & $9 / 21$ & A ICU & $\mathrm{R}$ & $\mathrm{R}$ & 1 & $\mathrm{R}$ & 1 & $\mathrm{R}$ & 1 & 2 & 2 & RESP \\
\hline 26 & $65 \mathrm{~F}$ & $11 / 9$ & C ICU & $\mathrm{R}$ & $\mathrm{R}$ & $\mathrm{R}$ & $\mathrm{R}$ & $\mathrm{R}$ & $\mathrm{R}$ & 1 & 2 & 3 & RESP \\
\hline 27 & $71 M$ & $9 / 6$ & D WARD & S & 1 & 1 & S & S & $\mathrm{R}$ & $\mathrm{R}$ & & 2 & URINE \\
\hline 28 & $45 \mathrm{~F}$ & $9 / 16$ & C ICU & S & $\mathrm{s}$ & $\mathrm{R}$ & $\mathrm{R}$ & s & $\mathrm{R}$ & $\mathrm{R}$ & & 2 & RESP \\
\hline 29 & $50 \mathrm{M}$ & $3 / 11$ & A ICU & $\mathrm{R}$ & $\mathrm{R}$ & $\mathrm{R}$ & $\mathrm{R}$ & $\mathrm{R}$ & $\mathrm{R}$ & $\mathrm{R}$ & 0.5 & 3 & URINE \\
\hline 30 & $61 \mathrm{~F}$ & $8 / 26$ & D ICU & $\mathrm{R}$ & $\mathrm{R}$ & $\mathrm{R}$ & $\mathrm{R}$ & $\mathrm{R}$ & $\mathrm{R}$ & $\mathrm{R}$ & 1.5 & 2 & BLOOD \\
\hline 31 & $78 \mathrm{M}$ & $11 / 10$ & A WARD & $\mathrm{S}$ & $\mathrm{R}$ & 1 & $\mathrm{R}$ & $\mathrm{s}$ & $\mathrm{R}$ & $\mathrm{R}$ & 0.5 & 2 & URINE \\
\hline
\end{tabular}

Study isolates (plus WC-31, which was isolated from a patient on Hospital Day 1 after transfer from an outside hospital). Legend WC = Weill Cornell; PT = Patient age and gender; Date: All dates are from 2008; Floor: Each letter represents a different hospital floor; Antimicrobial susceptibility to the various agents was determined following CLSI guidelines, or the Mean Inhibitory Concentration is reported directly for polymyxin B and tigecycline ( $\mu \mathrm{g} / \mathrm{mL}$ ): A/S: ampicillin-sulbactam, Carb: imipenem or meropenem; Cef: cefepime; Gent; gentamicin; Amik: amikacin; S/T: sulfamethoxazole-trimethoprim; Levo: levofloxacin; PM: polymyxin B; Tig: tigecycline; Source: Resp $=$ respiratory. Susceptibility interpretation based on 2008 CLSI cutoff points.

doi:10.1371/journal.pone.0028566.t001

\section{Molecular Epidemiology}

Rep-PCR testing showed that 23 of 30 (77\%) study isolates were closely related with similarity scores over $90 \%$ (Figure 1). This prevalent endemic strain of 23 isolates, hereafter referred to as the "prevalent strain", was geographically widespread and cultured from patients in 7 ICUs and 6 non-ICU nursing units. The isolate obtained from a patient transferred from a Brooklyn hospital (WC31 , was highly similar to the prevalent strain. MLST of a one prevalent strain isolate (WC-9) was consistent with ST-2, a member of Worldwide Clone II [15].

Seven additional isolates were not closely related to the prevalent group, or to each other, hereafter referred to as "sporadic isolates". One of the seven (14.3\%) sporadic isolates was MDR, compared with 20 of $23(87 \%)$ prevalent strain isolates.
Prevalent strain isolates were thus more likely to be MDR than the sporadic isolates (two tail Fisher's Exact $\mathrm{p}=0.001$ ).

\section{Integron Assessment of Prevalent A. baumannii}

Amplification of the integron variable region showed that all prevalent strain isolates contained a common 550 base pair product (GenBank \# JF309278, Figure 2). In contrast, the sporadic isolates contained products of varying sizes (data not shown), though two also contained an identically sequenced 550 base pair product. BLAST sequence analysis of the 550 base pair integron variable region showed $100 \%$ homology with a nonintegron-associated $A$. baumannii-associated sequence, which included a portion of a glucose-sorbosone dehydrogenase encoding gene (gb|CP000863.1). The same PCR sequence was amplified 


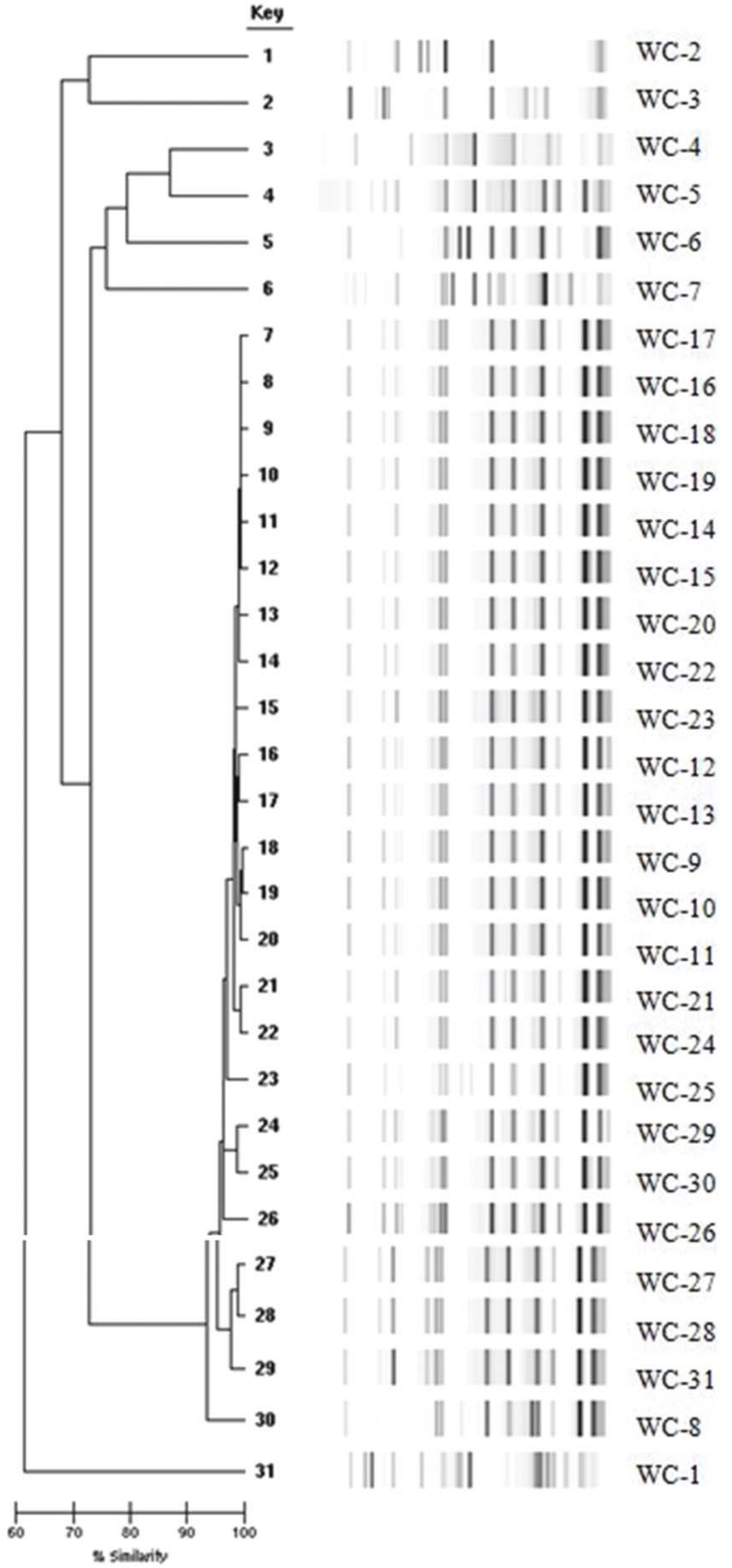


Figure 1. Rep-PCR Analysis of Study Isolates. Isolates with more than $90 \%$ similarity were considered related. Study isolates (WC 1-30) are shown, plus one isolate (WC-31) isolated on hospital day 1 after transfer from an outside hospital. Results show the majority of the study isolates are closely related.

doi:10.1371/journal.pone.0028566.g001

using alternative, non-overlapping Class I integron primers [7], confirming that this sequence was integron-associated in the study isolates.

\section{Metabolome Analysis}

Metabolic characteristics of both prevalent strain and sporadic isolates were compared by global metabolomic analysis and targeted analysis of individual metabolites. Biological replicates of three prevalent strain isolates (WC-9, WC-11, and WC-18) and 3 sporadic isolates (WC 1, WC-5, and WC-6) were selected and cultured using glucose as the sole carbon source. Acinetobacter species are known to metabolize glucose through the EntnerDoudoroff pathway [16]. The prevalent strain isolates and sporadic isolates did not differ in metabolites of glucose oxidation or the Entner-Doudoroff pathway (Figure 3). Finally, we compared the global metabolome profiles of prevalent strain isolates and sporadic isolates using Principle Components Analysis and Hierarchical Clustering Analysis. No significant differences in the global metabolite profiles of prevalent strain isolates and sporadic isolates were noted (Figure 3).

\section{Discussion}

A. baumannii is now endemic throughout NYG [4]. A limited number of circulating A. baumannii strains, some from multiple institutions and other from single sites, have been noted in previous studies [17-20]. The findings from our study, add to existing knowledge by showing the geographically wide distribution of a prevalent strain within one hospital during a period when no hospital-wide outbreak was suspected. The prevalent strain is a member of the expanding Worldwide Clone II, formerly known as European Clone II [15]. Persistence in hospital environments over prolonged periods appears to be a characteristic of successful $A$. baumannii strains $[21,22]$.

The prevalent $A$. baumannii strain contained a unique Class I integron sequence. This sequence was also amplified from two sporadic isolates, suggesting horizontal transfer. Integrons have been associated with prevalent $A$. baumannii strains [23], increased resistance to antibiotics [24], and persistence in hospital environments [25]. The significance of the partial glucose sorbosone dehydrogenase gene sequence encoded in the prevalent integron remains to be elucidated.

It is unclear why some isolates of $A$. baumannii become epidemic or endemic, and others remain sporadic. The virulence and persistence mechanisms of $A$. baumannii are only beginning to become understood [26], though significant genomic differences in A. baumannii isolates that are associated with human, environmental, or lice host environments have been noted [27]. Epidemic $A$. baumannii are able to survive on dry surfaces for prolonged periods in hospital environments, but do not persist longer than sporadic A. baumannii [28]. Antibiotic resistance itself is associated with increased persistence of some A. baumannii strains compared with more susceptible strains [29], though the relative contribution of antibiotic resistance and other unspecified virulence factors remains unknown. We found no significant metabolic differences between a limited number of tested sporadic isolates and prevalent strain isolates. While varying environmental conditions may lead to differences in isolates [30], our results are consistent with a primary role for antibiotic resistance itself as a marker for $A$. baumannii strains with increased potential to persist in hospitals. Furthermore, in contrast to previous metabolomic analysis of methicillin-susceptible and -resistant Staphylococcus aureus, as well as vancomycin-susceptible and -resistant Enterococcus faecium, the metabolic profiles of the isolates did not segregate by antibiotic resistance type [12]. This raises the question of whether antibiotic resistance in $A$. baumannii requires less metabolomic adaptation (or "cost") compared with these gram-positive organisms. Metabolomic characteristics of $A$. baumannii have been investigated [31], but strain to stain metabolomic variability had not been described to our knowledge. Further study is needed to clarify the metabolic, proteomic, and genomic differences associated with prevalent and sporadic MDR A. baumannii.
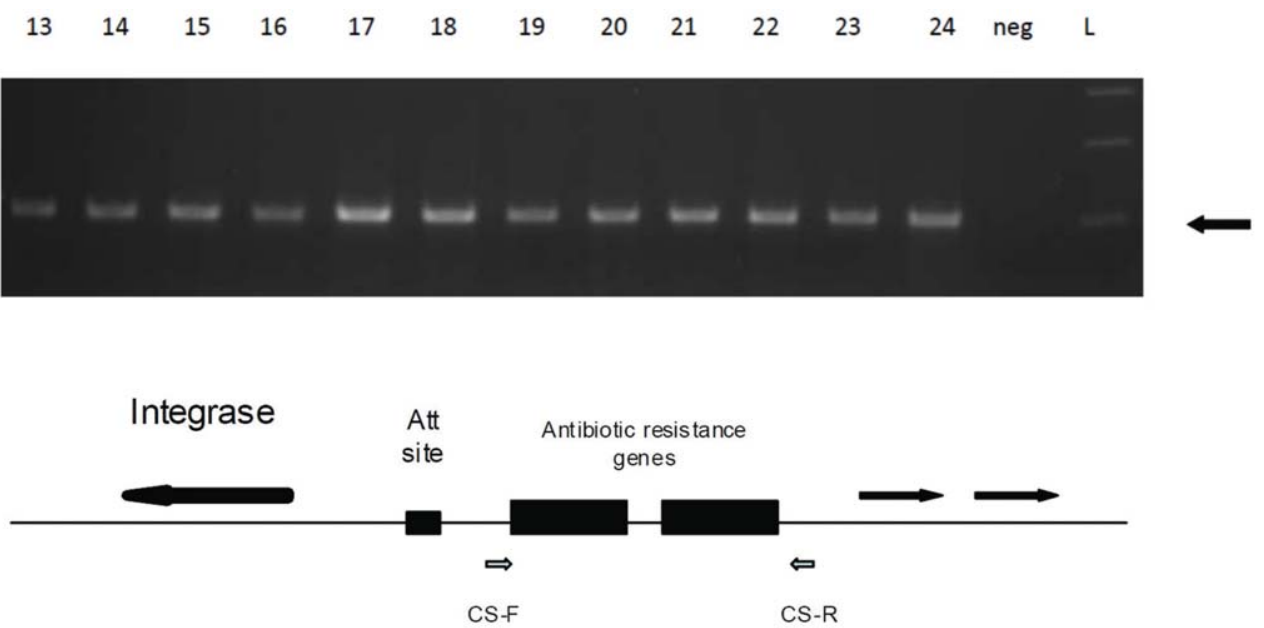

Figure 2. Integron Content in Select Study Isolates. Integron PCR gene products of WC 13-24 are shown. The block arrow marks the 550 base pair PCR product of the prevalent strain A. baumannii. The water control is labeled "neg." The cartoon shows the layout of a typical Class I integron. The PCR primers are presented by CS-F and CS-R.

doi:10.1371/journal.pone.0028566.g002 

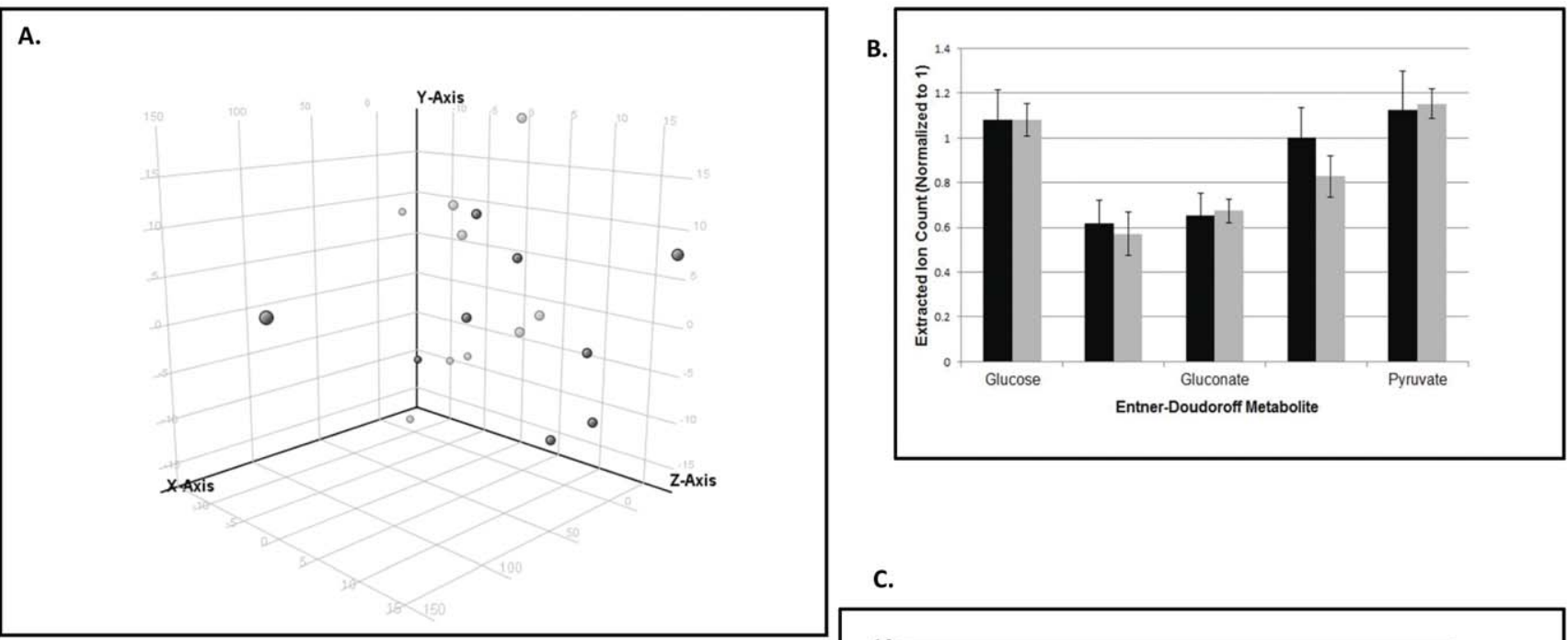

C.

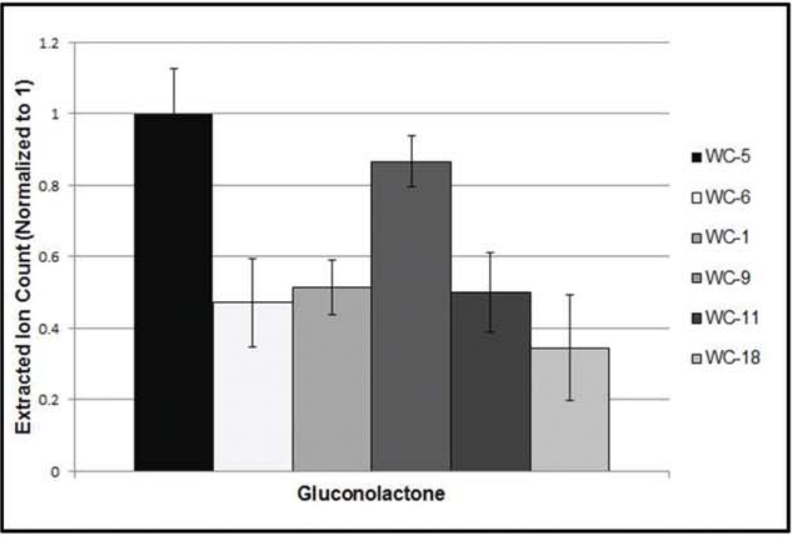

Figure 3. Metabolomic Analysis of Prevalent Endemic and Sporadic A. baumannii Isolates. (A) Principal components analysis of prevalent endemic (black circles) and sporadic (gray circles) isolates (in which the metabolomic profile of each isolate is represented as a single point on a 3-D axis) shows significant overap, consistent with similar metabolic profiles. (B) Targeted analysis of intermediates of the Entner-Doudoroff (ED) pathway shows no change in abundance of ED metabolites in sporadic (gray) vs prevalent endemic (black) isolates. (C) Abundance of gluconolactone (a representative intermediate in the ED pathway) did not differ significantly between sporadic isolates (WC-1, WC-5, and WC-6) and prevalent endemic isolates (WC-9, WC-5, and WC-6).

doi:10.1371/journal.pone.0028566.g003

The study has several limitations. We studied a non-random convenience sample of isolates selected to represent hospital-wide locations, which raises the potential of selection bias. Though Rep-PCR was not done on the available but excluded isolates, antibiogram and integron analysis suggest most excluded isolates belonged to the prevalent strain (data not shown). Only a subset of the total $A$. baumannii isolates from the hospital were collected over the study period, which may introduce additional bias if the collected isolates differed in any way from the hospital wide isolates. However, the antibiotic susceptibility profile of the study isolates was similar to that of isolates from both ICU and nonICU nursing units, suggesting that study isolates were representative of the hospital-wide A. baumannii strains. Our study period was only nine months and, thus, it is possible that we detected an unrecognized outbreak, rather than an endemic period, although the incidence and prevalence of $A$. baumannii had been stable for three years at the time of the study (data not shown). It is also possible that a longer sampling period would have uncovered additional prevalent strains of $A$. baumannii, such as those detected elsewhere in NYG [18]. Ongoing molecular epidemiologic surveillance of cultured isolates may further clarify the long term dynamics of the prevalent endemic A. baumannii strain within the hospital. Finally, isolates closely related by Rep-PCR may evolve clinically important differences, such as the differences in antimicrobial susceptibility testing results seen within the prevalent strain. Unrelated isolates may exchange genetic information and potentially share traits, highlighted by the finding of the prevalent strain integron in two of the sporadic isolates.

This study characterized the A. baumannii isolates from a NYG hospital. A previously unsuspected hospital-wide prevalent strain of multidrug-resistant $A$. baumannii was uncovered. The prevalent strain contained a novel integron cassette, though the functional significance of this finding requires further study. Metabolomic analysis did not segregate the prevalent strain from sporadic isolates. Identification of environmental, patient, and bacteriaspecific features associated with the development of persistent $A$. baumannii strains will aid efforts to combat this emerging pathogen.

\section{Acknowledgments}

Portions of this research were presented as poster abstracts at the $47^{\text {th }}$ Annual Infectious Disease Society of American meeting (Poster 816) in Philadelphia, PA, in October 2009, and at the National Clinical and Translational Research Education Annual Meeting (Poster 109) in Washington D.G., in April 2009. 


\section{Author Contributions}

Conceived and designed the experiments: SAW KYR ANS SGJ DHL. Performed the experiments: SAW ANS EAA BE MB. Analyzed the data:

\section{References}

1. Peleg A, Seifert H, Paterson D (2008) Acinetobacter baumannii: emergence of a successful pathogen. Clin Microbiol Rev 21: 538-582.

2. Gaynes R, Edwards J (2005) Overview of nosocomial infections caused by gramnegative bacilli. Clin Infect Dis 41: 848-854.

3. Talbot G, Bradley J, Edwards JJ, Gilbert D, Scheld M, et al. (2006) Bad bugs need drugs: an update on the development pipeline from the Antimicrobial Availability Task Force of the Infectious Diseases Society of America. Clin Infect Dis 42: 657-668.

4. Morgan D, Weisenberg S, Augenbraun M, Calfee D, Currie B, et al. (2009) Multidrug-resistant Acinetobacter baumannii in New York City - 10 years into the epidemic. Infect Control Hosp Epidemiol 30: 196-197.

5. Lévesque G, Piché L, Larose C, Roy P (1995) PCR mapping of integrons reveals several novel combinations of resistance genes. Antimicrob Agents Chemother 39: 185-191.

6. Martinez-Freijo P, Fluit A, Schmitz F, Grek V, Verhoef J, et al. (1998) Class I integrons in Gram-negative isolates from different European hospitals and association with decreased susceptibility to multiple antibiotic compounds. J Antimicrob Chemother 42: 689-696.

7. Barlow R, Pemberton J, Desmarchelier P, Gobius K (2004) Isolation and characterization of integron-containing bacteria without antibiotic selection. Antimicrob Agents Chemother 48: 838-842.

8. Turton J, Woodford N, Glover J, Yarde S, Kaufmann M, et al. (2006) Identification of Acinetobacter baumannii by detection of the blaOXA-51-like carbapenemase gene intrinsic to this species. J Clin Microbiol 44: 2974-2976.

9. Saeed S, Fakih M, Riederer K, Shah A, Khatib R (2006) Interinstitutional and intrainstitutional transmission of a strain of Acinetobacter baumannii detected by molecular analysis: comparison of pulsed-field gel electrophoresis and repetitive sequence-based polymerase chain reaction. Infect Control Hosp Epidemiol 27: 981-983.

10. Tomaras A, Flagler M, Dorsey G, Gaddy J, Actis L (2008) Characterization of a two-component regulatory system from Acinetobacter baumannii that controls biofilm formation and cellular morphology. Microbiology 154: 3398-3409.

11. Brauer M, Yuan J, Bennett B, Lu W, Kimball E, et al. (2006) Conservation of the metabolomic response to starvation across two divergent microbes. Proc Natl Acad Sci U S A 103: 19302-19307.

12. Weisenberg S, Butterfield T, Fischer S, Rhee K (2009) Suitability of silica hydride stationary phase, aqueous normal phase chromatography for untargeted metabolomic profiling of Enterococcus faecium and Staphylococcus aureus. J Sep Sci 32: 2262-2265.

13. Bradford M (1976) A rapid and sensitive method for the quantitation of microgram quantities of protein utilizing the principle of protein-dye binding. Anal Biochem 72: 248-254

14. Sana T, Roark J, Li X, Waddell K, Fischer S (2008) Molecular formula and METLIN Personal Metabolite Database matching applied to the identification of compounds generated by LC/TOF-MS. J Biomol Tech 19: 258-266.

15. Higgins P, Dammhayn C, Hackel M, Seifert H (2010) Global spread of carbapenem-resistant Acinetobacter baumannii. J Antimicrob Chemother 65: $233-238$.

16. Juni E (1978) Genetics and physiology of Acinetobacter. Annu Rev Microbiol 32: 349-371.
SAW KYR ANS EAA MB LS DHL SGJ. Contributed reagents/ materials/analysis tools: KYR SAW ANS SGJ MB EAA. Wrote the paper: SAW SGJ ANS LS EA KYR.

17. Jones R, Deshpande L, Fritsche T, Sader H (2004) Determination of epidemic clonality among multidrug-resistant strains of Acinetobacter spp. and Pseudomonas aeruginosa in the MYSTIC Programme (USA, 1999-2003). Diagn Microbiol Infect Dis 49: 211-216.

18. Quale J, Bratu S, Landman D, Heddurshetti R (2003) Molecular epidemiology and mechanisms of carbapenem resistance in Acinetobacter baumannii endemic in New York City. Clin Infect Dis 37: 214-220.

19. Landman D, Butnariu M, Bratu S, Quale J (2009) Genetic relatedness of multidrug-resistant Acinetobacter baumannii endemic to New York City. Epidemiol Infect 137: 174-180.

20. Landman D, Bratu S, Kochar S, Panwar M, Trehan M, et al. (2007) Evolution of antimicrobial resistance among Pseudomonas aeruginosa, Acinetobacter baumannii and Klebsiella pneumoniae in Brooklyn, NY. J Antimicrob Chemother 60: 78-82.

21. Baang JH, Axelrod P, Decker BK, Hujer AM, Dash G, et al. (2011) Longitudinal epidemiology of multidrug-resistant (MDR) Acinetobacter species in a tertiary care hospital. Am J Infect Control.

22. Runnegar N, Sidjabat H, Goh HM, Nimmo GR, Schembri MA, et al. (2010) Molecular epidemiology of multidrug-resistant Acinetobacter baumannii in a single institution over a 10-year period. J Clin Microbiol 48: 4051-4056.

23. Lee Y, Huang L, Chen T, Siu L, Fung C, et al. (2009) Gene cassette arrays, antibiotic susceptibilities, and clinical characteristics of Acinetobacter baumannii bacteremic strains harboring class 1 integrons. J Microbiol Immunol Infect 42: 210-219.

24. Huang L, Chen T, Lu P, Tsai C, Cho W, et al. (2008) Dissemination of multidrug-resistant, class 1 integron-carrying Acinetobacter baumannii isolates in Taiwan. Clin Microbiol Infect 14: 1010-1019.

25. Oh J, Kim K, Jeong Y, Cho J, Park J, et al. (2002) Epidemiological typing and prevalence of integrons in multiresistant Acinetobacter strains. APMIS 110: 247-252.

26. Gordon N, Wareham D (2010) Multidrug-resistant Acinetobacter baumannii: mechanisms of virulence and resistance. Int J Antimicrob Agents 35: 219-226.

27. Fournier P, Vallenet D, Barbe V, Audic S, Ogata H, et al. (2006) Comparative genomics of multidrug resistance in Acinetobacter baumannii. PLoS Genet 2: e7.

28. Jawad A, Seifert H, Snelling A, Heritage J, Hawkey P (1998) Survival of Acinetobacter baumannii on dry surfaces: comparison of outbreak and sporadic isolates. J Clin Microbiol 36: 1938-1941.

29. Rodríguez-Baño J, Cisneros J, Fernández-Cuenca F, Ribera A, Vila J, et al. (2004) Clinical features and epidemiology of Acinetobacter baumannii colonization and infection in Spanish hospitals. Infect Control Hosp Epidemiol 25: 819-824.

30. Soares N, Cabral M, Gayoso M, Mallo S, Rodriguez-Velo P, et al. (2010) Associating growth-phase-related changes in the proteome of Acinetobacter baumannii with increased resistance to oxidative stress. J Proteome Res.

31. Kim HU, Kim TY, Lee SY (2010) Genome-scale metabolic network analysis and drug targeting of multi-drug resistant pathogen Acinetobacter baumannii AYE. Mol Biosyst 6: 339-348. 